\title{
The expression of the $C E A C A M 19$ gene, a novel member of the CEA family, is associated with breast cancer progression
}

\author{
KLEITA MICHAELIDOU $^{1}$, ALEXANDROS TZOVARAS ${ }^{2}$, IOANNIS MISSITZIS ${ }^{3}$, \\ ALEXANDROS ARDAVANIS $^{2}$ and ANDREAS SCORILAS ${ }^{1}$ \\ ${ }^{1}$ Department of Biochemistry and Molecular Biology, University of Athens, 15701 Athens; \\ ${ }^{2}$ First Department of Oncology, and ${ }^{3}$ Department of Breast Cancer Surgery, \\ St. Savvas Anticancer Hospital, 11522 Athens, Greece
}

Received July 13, 2012; Accepted September 10, 2012

DOI: 10.3892/ijo.2013.1860

\begin{abstract}
Breast cancer (BC) continues to affect the lives of millions of women worldwide. Several members of the carcinoembryonic antigen-related cell adhesion molecule (CEACAM) subfamily are involved in tumor progression. Notably, the CEACAM subfamily harbors the already established cancer biomarker CEA, as well as other potential molecular markers. CEACAM19, a recently identified gene belonging to CEACAM subfamily, was discovered and cloned by members of our research group. The present study analyzes, quantitatively, the expression of CEACAM19 and evaluates its clinical relevance in BC. Total RNA was extracted from 143 cancerous and 89 normal adjacent breast tissue specimens. Following reverse transcription, quantitative analysis of CEACAM19 mRNA expression levels was performed via real-time PCR and the comparative $\mathrm{C}_{\mathrm{t}}\left(2^{-\Delta \Delta \mathrm{C} t}\right)$ method. CEACAM19 expression and detailed clinicopathological data were used for extensive biostatistical analyses. CEACAM19 was found to be overexpressed in breast cancer tissue specimens compared to normal tissue counterparts $(\mathrm{p}=0.013)$. CEACAM19 mRNA expression status was also associated with clinicopathological features indicative of aggressive behavior and poor prognosis in BC, such as high tumor grade $(\mathrm{p}=0.031)$ and high Ki67 proliferative index ( $\mathrm{p}=0.038)$. A significant negative association was documented between CEACAM19 expression and tumor ER status ( $\mathrm{p}=0.018)$ as well as patients' menopausal state $(\mathrm{p}=0.016)$. Our results suggest that $C E A C A M 19$ mRNA expression represents a promising, novel and clinically useful tissue biomarker for breast cancer management.
\end{abstract}

Correspondence to: Dr Andreas Scorilas, Department of Biochemistry and Molecular Biology, Faculty of Biology, University of Athens, Panepistimiopolis, 15701 Athens, Greece

E-mail: ascorilas@biol.uoa.gr

Key words: CEA-related cell adhesion molecules, CEA, CEACAM19, CEAL1, breast cancer, molecular tumor markers

\section{Introduction}

Breast cancer (BC), is the most commonly occurring malignancy in females and it continues to impose a major health burden globally. Despite the notable improvements in early diagnosis and the development of more effective therapeutic strategies, BC remains the leading cause of cancer-related mortality (1), with the vast majority of these deaths attributed to recurrent or metastatic disease (2). The development of metastasis requires interactions among breast cells and tumor microenvironment components, and implicates a variety of proteolytic enzymes, growth factors and cell adhesion molecules (3).

The carcinoembryonic antigen-related cell adhesion molecule (CEACAM) gene subfamily, belongs to the carcinoembryonic antigen (CEA) gene family; which in turn is a member of the immunoglobulin superfamily (IgSF) (4). In humans, the CEACAM subfamily members are involved in a variety of homotypic and/or heterotypic intercellular-adhesion and intracellular signaling events $(5,6)$, that govern several key biological processes, such as cell adhesion, cell growth, differentiation, immune response, cellular recognition, apoptosis and angiogenesis (7-10).

Apart from their physiological functions, recent studies demonstrate that the expression and/or function of CEACAM subfamily members are often deregulated in tumors, suggesting that they play an instrumental role in tumorigenesis, invasion and metastasis (9,11-13). Indeed, CEA (encoded by the CEACAM5 gene), and the closely related family member $C E A C A M 6$ are frequently found to be overexpressed in a majority of carcinomas $(14,15)$, and their overexpression is often associated with enhanced metastatic potential and, thus, with poor prognosis (11,12,16-18). On the contrary, CEACAM1 expression is usually reported to be downregulated in several tumor types, such as breast, prostate and colorectal cancer (8). Due to their differential expression in cancer and their documented tumorigenic functions $(4,8)$, many CEACAM members may possess clinical utility as prognostic/predictive markers for a panel of human malignancies. In particular, this notion is underscored by the routine clinical use of CEA serum levels in the prognosis, early detection of recurrence and follow-up of patients with breast, colorectal, or lung cancer (19). 
CEACAM19 gene, previously known as CEA-like gene 1 (CEAL1), was recently discovered and cloned by members of our research group. At the mRNA level, CEACAM19 is constitutively expressed in a wide range of normal tissues. However, the exact nature of its biological function remains to be fully elucidated. A preliminary study showed that CEACAM19 is upregulated, at the mRNA level, in ovarian and breast tumors. Interestingly, CEACAM19 overexpression was observed in clinically highly aggressive ovarian tumors suggesting that it could serve as a new cancer biomarker (20).

In the current study, we sought to analyze the expression of CEACAM19 and to further investigate its potential clinical significance in BC. Currently, BC management is mainly based on clinical and histological features such as tumor size, histological subtype and grade, as well as on molecular markers, such as estrogen receptor (ER), progesterone receptor $(\mathrm{PgR})$, and human epidermal growth factor receptor 2 (HER2) $(21,22)$. Nevertheless, all these parameters have a limited capacity to capture the great variability of biological and clinical behavior of breast carcinomas (23). Consequently, since BC is an extraordinarily heterogeneous disease entity (24), it is increasingly apparent that there is a great need for the identification and implementation of additional and more reliable tumor molecular biomarkers for early and effective diagnosis, prognosis and prediction of treatment outcome in $\mathrm{BC}$ patients. Here, we provide the first evidence that CEACAM19 gene expression analysis may provide important clinical information for patients suffering from BC.

\section{Materials and methods}

Collection of breast tissue samples and clinical data. Breast tumor samples $(\mathrm{n}=143)$ and matched non-malignant tissue sections $(n=89)$ were obtained from patients with breast carcinoma, who had undergone surgical treatment at the 'Saint Savvas' Anticancer Hospital of Athens, between February 2010 and March 2011. Each malignant and corresponding normal tissue sample was divided into two pieces. One of these was snap-frozen in liquid nitrogen immediately after the surgical resection and stored at $-80^{\circ} \mathrm{C}$ until the relevant assays were performed, and the second was histopathologically characterized in order to confirm the presence of malignancy.

A detailed database, containing clinical and pathological information concerning each patient was also provided for statistical analysis. None of the patients had received preoperative treatment. The age range was from 31 to 89 years with a median of 60 years. Tumor sizes ranged from 0.5 to $8.5 \mathrm{~cm}$ with a median of $2.4 \mathrm{~cm}$. Clinical staging was performed according to the Tumor-Node-Metastasis (TNM) classification system and histological grade was determined according to the Bloom-Scarff-Richardson grading system. ER, PgR and HER2 receptor status and Ki67 labeling index (percentage of Ki67 positive cancer nuclei) were determined by immunohistochemistry (IHC). ER and PgR immunostaining results were reported using a semi-quantitative immunohistochemical score (Hscore) which incorporated both the staining intensity $(i)$ and the corresponding percentage of positive stained cells $(\mathrm{P} i)(25)$. The Hscore is given by the equation Hscore $=\Sigma(\mathrm{P} i * i / 100)$ and ranges from 0 to 3 . The clinicopathological data obtained from the pathology report such as age, tumor size, hormone receptors' Hscore and Ki67 proliferation index, are summarized in Table I.

All of the research procedures that took place during the course of our study were performed according to the ethical standards of the 1975 Declaration of Helsinki, as revised in 2008, and were approved by the institutional review board of 'Saint Savvas' Anticancer Hospital. Moreover, written informed consent was obtained from all BC patients participating in the study.

Total RNA extraction, RNA quality evaluation and cDNA synthesis. Specimens of 50-100 mg were cut from the frozen breast tissue samples, with a prechilled scalpel without thawing, and pulverized in liquid nitrogen. Then, the resulting homogeneous powder was dissolved in $1 \mathrm{ml}$ of TRI ${ }^{\circledR}$ Reagent (Ambion Inc., Austin, TX, USA) and total RNA was extracted according to the manufacturer's protocol. All RNA samples were preserved with RNA-Storage solution (Ambion Inc.) and stored at $-80^{\circ} \mathrm{C}$ until use. The concentration and purity of RNA were determined spectrophotometrically at 260 and $280 \mathrm{~nm}$, while its integrity was assessed by agarose gel electrophoresis. Two micrograms of total RNA from each sample were reverse-transcribed into first-strand cDNA, in a $20 \mu \mathrm{l}$ reaction mixture, using M-MLV Reverse Transcriptase (Invitrogen, Life technologies, Carlsbad, CA, USA) and Oligo(dT) primers.

Quantitative real-time polymerase chain reaction ( $q R T-P C R)$. Gene specific primers were designed for HPRTI (hypoxanthine phosphoribosyltransferase 1 , housekeeping gene) and for CEACAM19, based on their published cDNA sequences in the NCBI Sequence database (GenBank accession nos. NM_000194.2 for HPRT1 and NM_020219.3 for CEACAM19), using the Primer Express software (Applied Biosystems, Foster City, CA, USA). The sequences of the HPRT1 primers were as follows: 5'-TGG AAA GGG TGT TTA TTC CTC AT-3' and 5'-ATG TAA TCC AGC AGG TCA GCA A-3' resulting in a 151 bp PCR amplicon, whereas the sequences of the CEACAM19 primers were: 5'-GAG GTC CAG GTA GCT GAA AAG A-3' and 5'-GGA TAC AGC CGA GCA CAA GA-3', generating a 222 bp PCR amplicon.

Real-time monitoring of the PCR reaction was performed using a 7500 real-time PCR System (Applied Biosystems, Inc.) and the SYBR-Green I chemistry (Fig. 1A). The reaction mixture consisted of $10 \mathrm{ng}$ of template cDNA, 5.0 $\mu \mathrm{l} \mathrm{KAPA}$ SYBR $^{\circledR}$ FAST qPCR Master mix (Kapa Biosystems, Woburn, MA, USA), $1.0 \mu$ of each gene-specific primer (final concentration $75 \mathrm{nM}$ each) and the final reaction volume was adjusted to $10.0 \mu \mathrm{l}$, with DEPC-treated water. The thermal protocol conditions were as follows: an initial step of polymerase activation at $95^{\circ} \mathrm{C}$ for $3 \mathrm{~min}$, followed by 40 cycles of denaturation at $95^{\circ} \mathrm{C}$ for $15 \mathrm{sec}$ and primer annealing and extension at $60^{\circ} \mathrm{C}$ for $1 \mathrm{~min}$. Each sample was amplified in triplicate, and the average $\mathrm{C}_{\mathrm{t}}$ values were calculated for the subsequent expression analysis. Following amplification, dissociation curves were generated for distinguishing the specific PCR products from non-specific products and/or any primer-dimers, through their unique melting temperatures (Tm) (Fig. 1C). Furthermore, in order to confirm the amplification specificity, the qRT-PCR products were electrophoresed on $3.0 \%$ (w/v) agarose gel and visualized, under UV light, after ethidium bromide staining (Fig. 1D). 
Table I. Distribution of numerical variables of the study in breast cancer patients.

Percentiles

\begin{tabular}{|c|c|c|c|c|c|c|c|}
\hline & & & & & & & \\
\hline Variables & Mean $\pm \mathrm{SE}^{\mathrm{a}}$ & Range & 10 & 25 & 50 & 75 & 90 \\
\hline $\begin{array}{l}C E A C A M 19 \text { (RQ units) } \\
\text { in cancer tissues }(\mathrm{n}=143)\end{array}$ & $0.542 \pm 0.071$ & $0.008-5.19$ & 0.008 & 0.019 & 0.182 & 0.708 & 1.52 \\
\hline $\begin{array}{l}C E A C A M 19 \text { (RQ units) } \\
\text { in non-cancer tissues }(\mathrm{n}=89)\end{array}$ & $0.462 \pm 0.098$ & $0.008-4.87$ & 0.008 & 0.008 & 0.008 & 0.464 & 1.45 \\
\hline Age (years) & $59.7 \pm 1.15$ & $31.0-89.0$ & 39.2 & 50.0 & 60.0 & 71.0 & 77.0 \\
\hline Tumor size $(\mathrm{cm})$ & $2.70 \pm 0.129$ & $0.50-8.50$ & 1.40 & 1.80 & 2.40 & 3.10 & 5.32 \\
\hline Ki67 proliferation index $(\%)$ & $13.5 \pm 1.21$ & $0.00-60.0$ & 1.00 & 3.00 & 10.0 & 20.0 & 30.0 \\
\hline $\mathrm{CEA}(\mathrm{ng} / \mathrm{ml})$ & $2.54 \pm 0.376$ & $0.20-31.1$ & 0.680 & 1.10 & 1.88 & 2.90 & 4.40 \\
\hline Estrogen receptor $(E R)^{c}$ & $1.29 \pm 0.100$ & $0.00-3.00$ & 0.00 & 0.01 & 1.30 & 2.10 & 3.00 \\
\hline Progesterone receptor $(\mathrm{PR})^{\mathrm{c}}$ & $0.765 \pm 0.086$ & $0.00-3.00$ & 0.00 & 0.00 & 0.100 & 1.67 & 2.50 \\
\hline
\end{tabular}

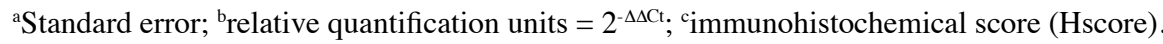

Gene expression analysis was performed using the comparative $C_{t}\left(2^{-\Delta \Delta C t}\right)$ method, to calculate the relative quantification units (RQ units) for each sample. HPRT1 served as an internal control gene for normalization purposes, whereas the human breast cancer cell line BT-474 was used as a calibrator allowing PCR comparison from distinct runs (26). The $\Delta C_{t}$ value represents the difference between the threshold cycle $\left(\mathrm{C}_{\mathrm{t}}\right)$ of the target gene (CEACAM19) and the $\mathrm{C}_{\mathrm{t}}$ of the corresponding endogenous reference gene (HPRT1) of a sample under study, while the $\Delta \Delta \mathrm{C}_{\mathrm{t}}$ value is the difference between the average $\Delta \mathrm{C}_{\mathrm{t}}$ value of an experimental sample and the average $\Delta \mathrm{C}_{\mathrm{t}}$ of the corresponding calibrator.

Statistical analysis. Our data were subjected to statistical analysis using the SPSS software program (SPSS Inc., Chicago, IL, USA). Differences between the relative expression levels of CEACAM19 obtained from matched normal and tumor compartments were tested using the Wilcoxon Signed Ranks test. Receiver Operating Characteristic curve (ROC) was constructed for CEACAM19 expression levels, by plotting sensitivity versus (1-specificity), and the area under the ROC curve (AUC) was analyzed by the Hanley and McNeil method. Logistic regression analysis was used to calculate the odds ratio that defines the relation between CEACAM19 expression and $\mathrm{BC}$ risk. Correlations between different variables were assessed by the Spearman correlation coefficient $\left(r_{s}\right)$. Furthermore, the $\mathrm{X}$-tile algorithm was applied in order to produce an optimal cutoff value for CEACAM19 (27), since there are no established cutoff points regarding its expression. Thus, an optimal cutoff point of $0.18 \mathrm{RQ}$ units was generated, which is equal to the 50th percentile. According to this cutoff value, tumors were categorized as CEACAM19-positive or CEACAM19-negative and associations between CEACAM19 expression status and other qualitative clinicopathological parameters were analyzed using the $\chi^{2}$ test or the Fisher's exact test, where appropriate. Patients' menopausal status was defined according to age as follows: premenopausal ( $<55$ years) and postmenopausal ( $>55$ years). The cutoff values for CEA and CA15.3 serum levels were $5.0 \mathrm{ng} / \mathrm{ml}$ and $27 \mathrm{U} / \mathrm{ml}$, respectively. ER and PgR status were considered as negative if the Hscore was below the minimum cutoff value of 0.35 and 0.25 , respectively. In case of HER2, IHC staining was categorized as follows: 0 , no staining; $1+$, weak staining; $2+$, complete membrane staining that is either non-uniform or weak in intensity; and $3+$, intense staining of $>30 \%$ of tumor cells. Regarding Ki67 labeling index, a cutoff value of $14 \%$ was used, as proposed by the recent Saint Gallen Consensus Conference Guidelines, to distinguish tumors with low $(<14 \%)$ and high ( $>14 \%)$ proliferative fraction (28). A P-value of $<0.05$ was considered as an indication of statistical significance.

\section{Results}

Validation of the comparative $C_{t}\left(2^{-\Delta \Delta C t}\right)$ method for CEACAM19 $m R N A$ quantification. A prerequisite for the application of the comparative $\mathrm{C}_{\mathrm{t}}\left(2^{-\Delta \Delta \mathrm{Ct}}\right)$ method is that the PCR amplification efficiencies of the target (CEACAM19) and the reference (HPRT1) gene are approximately equal and close to $100 \%$ (26). In order to determine PCR efficiencies for each gene, a validation experiment was carried out, in which $\mathrm{C}_{\mathrm{t}}$ values of CEACAM19 and HPRT1 were measured in serial dilutions of control cDNA prepared from total RNA from BT-474 breast cancer cells, over a 100 -fold range. A plot of $C_{t}$ values versus $\log$ of cDNA concentration was constructed for each gene and real-time PCR efficiencies (E) were calculated from the given slopes, according to the equation: $\mathrm{E}(\%)=\left(10^{(-1 / \mathrm{slope})}-1\right) \times 100$.

As illustrated in Fig. 1B, the slopes of HPRT1 and CEACAM19 plots, were similar (-3.288 and -3.268 , correspondingly), and the calculated PCR amplification efficiencies were 101.4\% (HPRT1) and $102.3 \%$ (CEACAM19), allowing the relative quantification by the application of the $2^{-\Delta \Delta \mathrm{Ct}}$ formula.

Analysis of CEACAM19 relative expression levels in breast tumors and non-malignant breast tissue sections. Expression of the CEACAM19 gene was observed in both cancerous and 
A

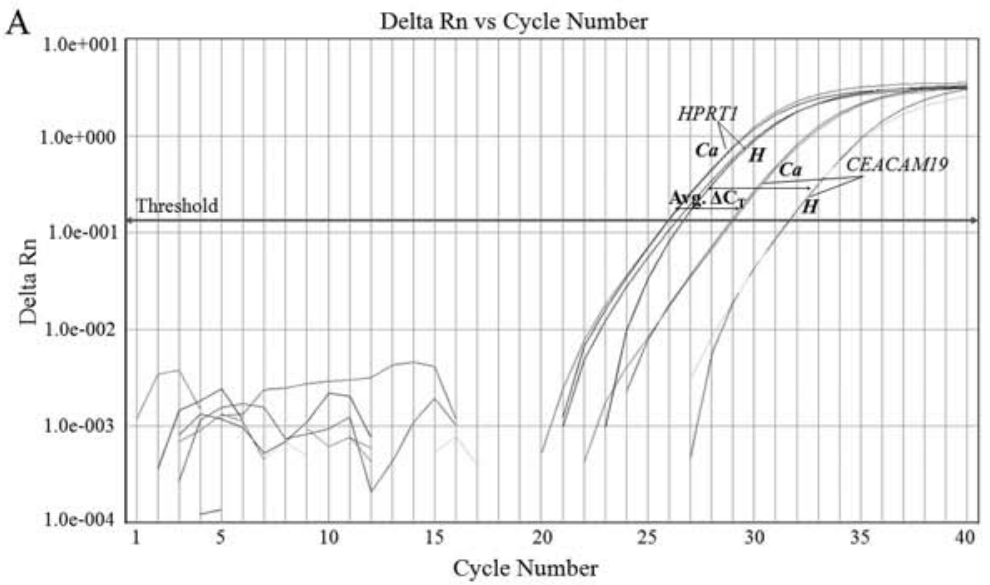

B

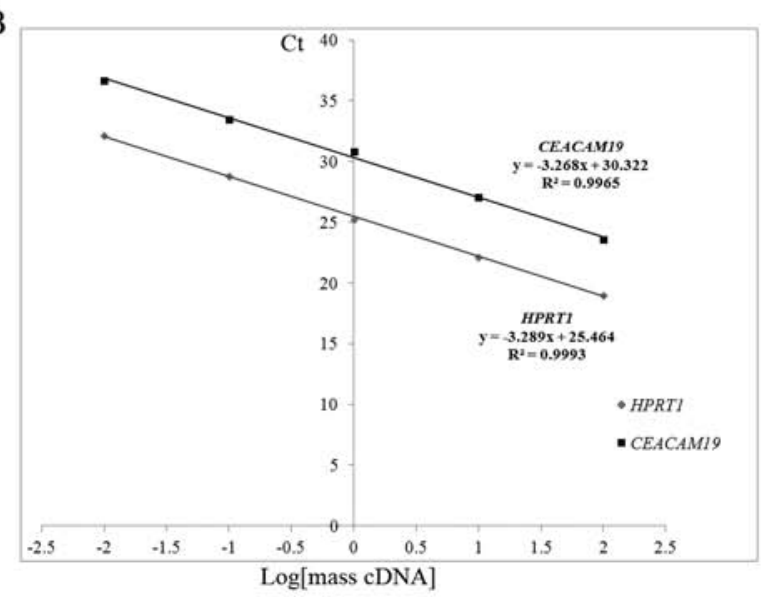

$\mathrm{C}$
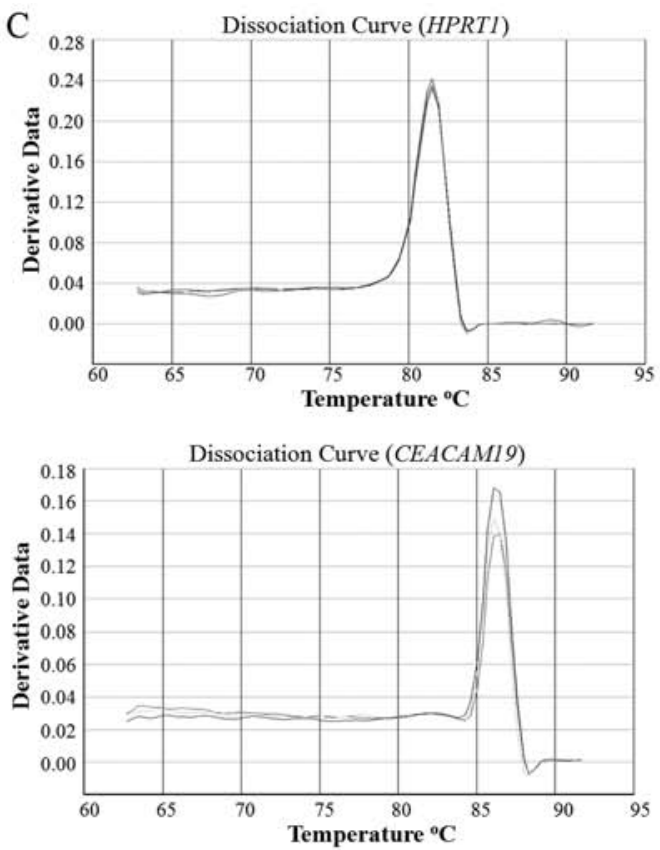
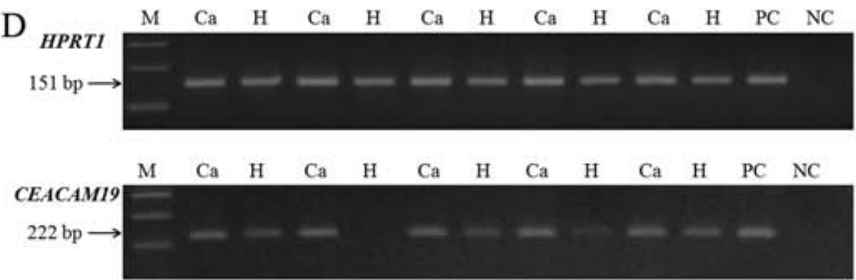

Figure 1. Relative quantification of the CEACAM19 expression via real-time PCR. (A) A representative amplification plot of CEACAM19 and $H P R T 1$, in a randomly selected pair of matched cancerous (Ca) and non-cancerous (H) breast tissue parts. (B) Validation of the comparative $\mathrm{C}_{\mathrm{t}}\left(2^{-\triangle \Delta C}\right)$ method, for the target $(C E A C A M 19)$ and the internal control (HPRT1) genes. (C) Dissociation curves of HPRT1 and CEACAM19 PCR products. (D) Agarose gel electrophoresis (3.0\% w/v) of $H P R T 1$ and CEACAM19 real-time PCR products in randomly selected breast tissue samples. M, molecular weight marker; PC, positive control; NC, negative control; $\mathrm{Ca}$, cancer tissue; part $\mathrm{H}$, matched non-malignant tissue part.

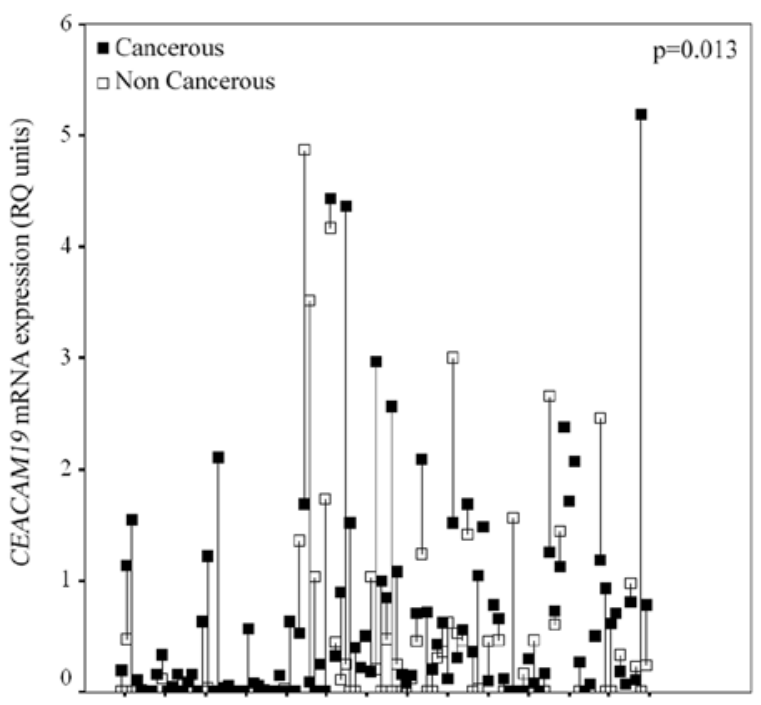

Breast Cancer Patients

Figure 2.CEACAM19 mRNA expression in paired breast tissue samples. Black boxes represent $C E A C A M 19$ expression in cancerous breast tissue sections and grey boxes in non-malignant tissue parts. P-value, calculated by Wilcoxon signed ranks test. non-neoplastic breast tissue samples. Interestingly, after examining CEACAM19 expression in the cohort of the 89 paired breast tissue samples, a statistically significant $(\mathrm{p}=0.013)$, CEACAM19 overexpression in cancerous breast tissue sections compared to their normal counterparts, was observed, in the majority of the patients. In more detail, CEACAM19 expression levels were higher in the cancerous tissue compared to the matched non-cancerous component in 59.55\% of the paired samples, whereas only $23.59 \%$ of the paired tissues showed lower CEACAM19 expression in the cancerous part compared to their matched normal counterpart (Fig. 2).

Furthermore, relative quantification units (RQ units) of CEACAM19 in cancerous specimens ranged from 0.008 to $5.19 \mathrm{RQ}$ units with a mean $( \pm \mathrm{SE})$ of $0.542( \pm 0.071)$. In non-cancerous breast tissue samples, CEACAM19 relative expression levels varied from 0.008 to 4.87 RQ units with a mean $( \pm$ SE) of $0.462( \pm 0.098)$. The median (50th percentile) value of CEACAM19 relative expression levels was found to be approximately 23 -fold higher in $\mathrm{BC}$ tissues (median: $0.182 \mathrm{RQ}$ units) compared to normal tissue specimens (median: 0.008 RQ units) (Table I). ROC curve analysis (Fig. 4), revealed a statistically significant $(\mathrm{p}=0.002)$ value of $C E A C A M 19$ expression in differentiating malignant from non-malignant 
Table II. Relationships between CEACAM19 expression status and clinicopathological variables.

\begin{tabular}{|c|c|c|c|c|}
\hline \multirow[b]{2}{*}{ Variable } & \multirow[b]{2}{*}{ Total } & \multicolumn{2}{|c|}{ No. of patients (\%) } & \multirow[b]{2}{*}{ P-value } \\
\hline & & $\begin{array}{c}\text { CEACAM19- } \\
\text { negative }^{\mathrm{a}}\end{array}$ & $\begin{array}{c}\text { CEACAM19- } \\
\text { positive }^{\mathrm{a}}\end{array}$ & \\
\hline Tumor grade & & & & $0.031^{\mathrm{b}}$ \\
\hline I & 7 & $6(85.7)$ & $1(14.3)$ & \\
\hline II & 86 & $46(53.5)$ & $40(46.5)$ & \\
\hline III & 34 & $12(35.3)$ & $22(64.7)$ & \\
\hline $\mathrm{X}$ & 16 & & & \\
\hline Tumor stage & & & & $0.971^{\mathrm{b}}$ \\
\hline I & 42 & $21(50.0)$ & $21(50.0)$ & \\
\hline II & 74 & $37(50.0)$ & $37(50.0)$ & \\
\hline III & 15 & $8(53.3)$ & $7(46.7)$ & \\
\hline $\mathrm{X}$ & 12 & & & \\
\hline Ki67 proliferative index & & & & $0.038^{c}$ \\
\hline Low proliferative fraction & 64 & $37(57.8)$ & $27(42.2)$ & \\
\hline High proliferative fraction & 48 & $18(37.5)$ & $30(62.5)$ & \\
\hline $\mathrm{X}$ & 31 & & & \\
\hline CEA & & & & $1.00^{\mathrm{c}}$ \\
\hline Negative & 83 & $41(49.4)$ & $42(50.6)$ & \\
\hline Positive & 4 & $2(50.0)$ & $2(50.0)$ & \\
\hline $\mathrm{X}$ & 56 & & & \\
\hline CA $15-3$ & & & & $0.470^{\mathrm{c}}$ \\
\hline Negative & 68 & $34(50.0)$ & $34(50.0)$ & \\
\hline Positive & 23 & $9(39.1)$ & $14(60.9)$ & \\
\hline $\mathrm{X}$ & 52 & & & \\
\hline ER-status & & & & $0.018^{\mathrm{c}}$ \\
\hline Negative & 46 & $16(34.8)$ & $30(65.2)$ & \\
\hline Positive & 84 & $48(57.1)$ & $36(42.9)$ & \\
\hline $\mathrm{X}$ & 13 & & & \\
\hline PgR-status & & & & $1.00^{\mathrm{c}}$ \\
\hline Negative & 68 & $34(50.0)$ & $34(50.0)$ & \\
\hline Positive & 63 & $32(50.8)$ & $31(49.2)$ & \\
\hline $\mathrm{X}$ & 12 & & & \\
\hline Menopausal status & & & & $0.016^{\mathrm{c}}$ \\
\hline Premenopausal & 33 & $10(30.3)$ & $23(69.7)$ & \\
\hline Postmenopausal & 106 & $59(55.7)$ & $47(44.3)$ & \\
\hline $\mathrm{X}$ & 4 & & & \\
\hline HER2 status & & & & $0.847^{\mathrm{b}}$ \\
\hline 0 & 71 & $35(49.3)$ & $36(50.7)$ & \\
\hline $1+$ & 19 & $9(47.4)$ & $10(52.6)$ & \\
\hline $2+$ & 13 & $8(61.5)$ & $5(38.5)$ & \\
\hline $3+$ & 19 & $9(47.4)$ & $10(52.6)$ & \\
\hline $\mathrm{X}$ & 21 & & & \\
\hline
\end{tabular}

$\mathrm{X}$, status unknown; acut-off point, $0.18 \mathrm{RQ}$ units, equal to the 50th percentile; ${ }^{\mathrm{b}}$ calculated by $\chi^{2}$ test; ${ }^{\mathrm{c}}$ calculated by Fisher's exact test.

breast tissues (AUC,0.622; 95\% CI=0.545-0.700). Additionally, logistic regression analysis demonstrated that CEACAM19 expression was significantly associated with $\mathrm{BC}$ risk, since an elevation in CEACAM19 expression levels is associated with increased risk of suffering from BC (odds ratio, 1.39, 95\% $\mathrm{CI}=1.03-1.86, \mathrm{p}=0.027$ ).
CEACAM19 expression status in BC tissues and its association with clinicopathological features of breast cancer patients. The median value of the CEACAM19 relative expression levels (0.18 RQ units), was adopted as an optimal cutoff point, in order to investigate the possible relationship between the CEACAM19 expression status of the tumors 


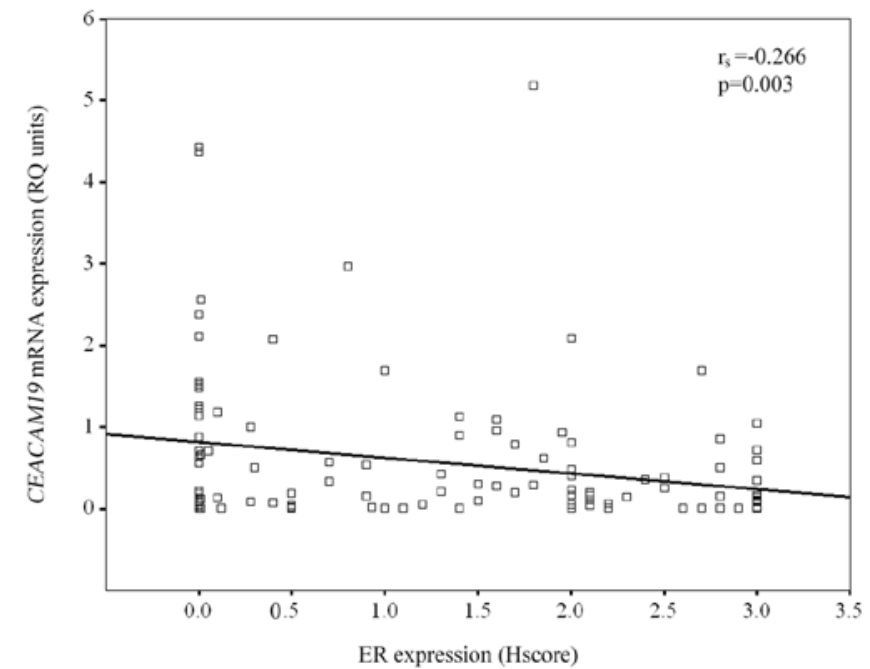

Figure 3. Correlation between CEACAM19 mRNA expression levels in breast tumors and ER expression (Hscore). $\mathrm{r}_{\mathrm{s}}$, Spearman correlation coefficient.

(CEACAM19-positive or CEACAM19-negative) with the clinical and pathological data obtained from the $\mathrm{BC}$ patients (Table II).

As far as the tumors' histological grade is concerned, CEACAM19 levels were significantly $(\mathrm{p}=0.031)$ elevated in poorly differentiated tumors (Grade III), compared to those of well and moderate differentiation states (Grade I/II). Specifically, CEACAM19-positivity was more often found in Grade III (64.7\%) tumors than in Grade I (14.3\%) and Grade II (46.5\%) tumors. Beside the histological grade, a statistically significant positive association between CEACAM19 expression status and Ki67 labeling index ( $\mathrm{p}=0.038)$, was also revealed. More precisely, $62.5 \%$ of the tumors with high proliferative fraction were found to be CEACAM19-positive, whereas only $42.2 \%$ of those with low proliferative fraction were detected with CEACAM19 expression levels above the adopted cutoff value. On the other hand, CEACAM19 expression status was not associated with tumor stage, HER2 status, and CEA or CA15.3 serum levels.

Furthermore, as indicated by our results, CEACAM19 mRNA expression status was negatively associated with the estrogen receptors as well as the patients' menopausal status, to a statistically significant degree. In particular, regarding ER-status, CEACAM19 mRNA levels were found to be significantly $(\mathrm{p}=0.018)$ higher in tumors with ER-negative staining, compared to ER-positive tumors. CEACAM19-positivity was more frequently found in ER-negative tumors (65.2\%) than in ER-positive tumors (42.9\%). The negative association between CEACAM19 expression and ER expression status was also supported by the calculated negative Spearman correlation coefficient $\left(r_{s}=-0.266 ; p=0.003\right)$ (Fig. 3). On the contrary, no significant association was observed between CEACAM19 expression and PgR status. In addition, CEACAM19-positivity was found significantly $(\mathrm{p}=0.016)$ more often in tumors derived from premenopausal women $(69.7 \%)$, than in those obtained from postmenopausal women (44.3\%).

Taken together, these data strongly suggest that higher CEACAM19 expression is associated with several indicators of aggressive tumor behavior and poor clinical outcome in

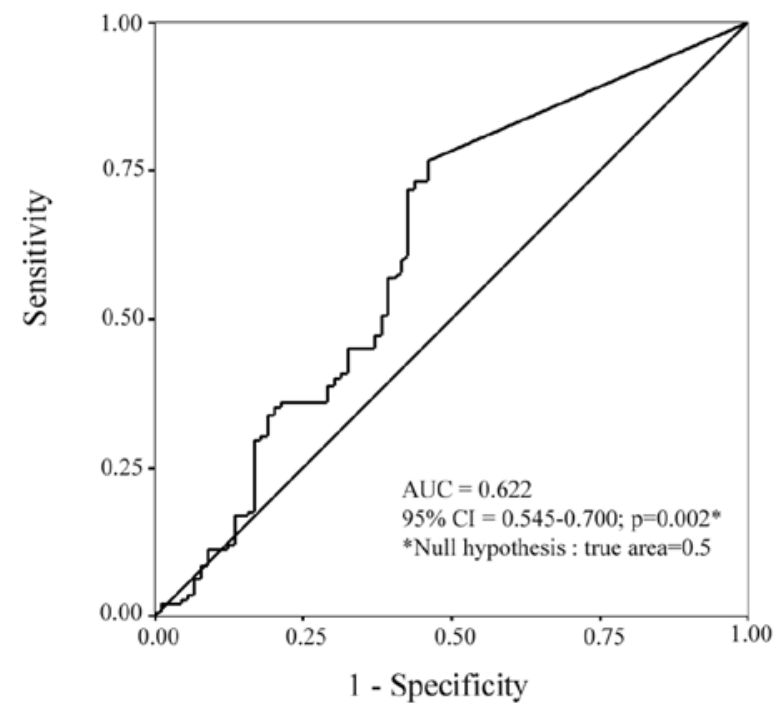

Figure 4. Receiver-operating characteristic (ROC) curve for CEACAM19 expression. CI, confidence interval; AUC, area under the curve.

BC patients, including high histological grade (Grade III), high tumor proliferative index (Ki67 labeling index), ER-negative status and patients' premenopausal state.

\section{Discussion}

Breast cancer (BC), the most common malignancy which affects the female population, constitutes a major cause of morbidity and mortality, globally. Despite extensive research efforts in the field of biomedical $\mathrm{BC}$ research, early diagnosis and management of $\mathrm{BC}$ patients, still face major challenges (1). Therefore, it is increasingly apparent that the identification of new and more reliable tumor molecular markers, which can be used either solely or in suitable combinations with other biomarkers and/or clinical parameters, can aid the differential diagnosis, accurate prognosis and treatment tailoring of $\mathrm{BC}$ patients. This approach could provide a clinically relevant solution in order to control this extremely heterogeneous disease.

Breast tumor biomarkers can be found among molecules that take part in key-processes often characterized as the hallmarks of cancer. Members of the CEACAM subfamily are known to be involved in various aspects of tumor progression and metastasis by affecting both intercellular adhesion and intracellular signaling $(4,8)$ and thus, represent one example of such molecules. Indeed, CEA, the prototypic member of the CEACAM subfamily, is one of the first known and most widely used tumor markers in clinical management of patients with colorectal, breast or lung cancer (19). In addition, other CEACAM members may also possess clinical utility as prognostic/predictive markers for a panel of human malignancies, including BC. Interestingly, CEACAM6 protein expression is an important predictor of subsequent invasive BC development, in patients with precancerous lesions (29) and of future recurrence in endocrine-resistant breast tumors (30). Additionally, altered splicing of CEACAM1 was observed in $\mathrm{BC}$ and aberrant expression of its splice variants in cancerous compared to normal breast tissue may have an important prognostic value for this malignancy (31). 
Given the above, we sought to analyze CEACAM19 mRNA expression in breast tumors and matched adjacent normal breast tissue sections. The objective of this study was to investigate whether CEACAM19 expression levels have a clinical value in the discrimination of cancerous from non-cancerous breast tissues and to further assess any possible relationship between CEACAM19 expression and clinicopathological variables of $\mathrm{BC}$ patients. To the best of our knowledge, this is the first study examining quantitatively CEACAM19 expression and its clinical value, in a large cohort of clinical breast tissue samples.

According to our data, CEACAM19 mRNA expression was detected in both cancerous and non-cancerous breast tissue specimens. However, in the cohort of the 89 paired breast tissue samples, CEACAM19 was significantly overexpressed $(\mathrm{p}=0.013)$ in the cancerous breast tissue compared to their matched normal counterparts. The majority of the paired samples (59.55\%) were found with higher CEACAM19 expression in cancer versus normal tissue, whereas only $23.59 \%$ of the paired tissues showed lower CEACAM19 expression in cancer compared to the normal tissue parts. Furthermore, the median value of CEACAM19 relative expression levels was approximately 23 -fold higher in $\mathrm{BC}$ tissues compared to normal tissue specimens (Table I). Additionally, ROC curve analysis revealed that CEACAM19 is differentially expressed, at a statistically significant degree $(\mathrm{p}=0.002)$, and can be used for the discrimination of malignant from non-malignant breast tissues (Fig. 4). Moreover, logistic regression analysis demonstrated that patients with high CEACAM19 expression levels were at increased risk of suffering from $\mathrm{BC}(\mathrm{p}=0.027)$. These results are in agreement with a preliminary study that demonstrated that $C E A C A M 19$ expression is lower in normal, compared to cancerous breast and ovarian tissue samples (20). Furthermore, our observation that $C E A C A M 19$ is overexpressed in $\mathrm{BC}$ tissue samples is consistent with the upregulation of other CEACAM family members in BC and in other carcinomas (12), and further suggests the possible involvement of the CEACAM19 molecule in breast tumor pathobiology.

In the present study, CEACAM19 expression was also scrutinized for its prognostic value, which could arise from any relationships with the clinicopathological data of the patients examined (Table II). Intriguingly, this analysis revealed a statistically significant positive association between CEACAM19 expression status with tumor grade $(\mathrm{p}=0.031)$. This finding suggests that $C E A C A M 19$ expression may provide valuable information for more detailed molecular discrimination between low- and high-grade tumors. Furthermore, our observation is in agreement with different studies, which showed that the expression of other subfamily members is often associated with tumor grade. In particular, in gastric cancer, CEACAM7 protein expression is more frequently found in poorly differentiated compared to well and moderately differentiated gastric carcinomas (32). Additionally, CEA and CEACAM6 expression in colorectal cancer correlates inversely with the degree of cellular differentiation (33). It is also possible that deregulated expression of CEACAM19 may disrupt cellular differentiation during tumor progression. This assumption is supported by earlier studies which provide evidence that deregulated overexpression of several CEACAM members, such as CEA and CEACAM6, are capable of inhibiting cellular differentiation in many cell types (33).
Another important finding of the present study is the statistically significant positive association $(\mathrm{p}=0.038)$ between CEACAM19 expression status with tumor Ki67 labeling index. This observation is consistent with the association of CEACAM19 expression with high histological grade, since high Ki67 protein expression is in consonance with higher tumor grade. Notably, high Ki67 proliferative index is an established indicator of aggressive tumor behavior and increased risk of relapse and death in BC patients (21). Therefore, this observation discloses another proof that CEACAM19 expression is associated with manifestations of poor prognosis and suggests that its upregulated expression may contribute to the aggressive nature of high Ki67 tumors by promoting cellular proliferation. Supporting this notion, a recent study has shown that CEACAM6 may act as an inducer of cellular proliferation in a subpopulation of A549 human lung cells, and its expression is also associated with Ki67-positive staining (13). Additionally, in accordance with our results, a different research group demonstrated that the expression of another member of the subfamily, CEACAM1, in pancreatic endocrine tumors is strongly associated with high Ki67 labeling index (34).

Another point to be addressed is the significant negative association between CEACAM19 expression status and the tumors' ER status ( $\mathrm{p}=0.018$ ), given that CEACAM19positivity, was more frequently found in ER-negative compared to ER-positive tumors. It is well known that ER-negative breast carcinomas are a distinct group of tumors with poor prognosis, due to their resistance to hormonal therapies (21). Thus, our findings give additional evidence that CEACAM19 is associated with poor prognosis. This raises the possibility that, CEACAM19 expression assessment, may serve as a clinically useful tool for predicting tumor response to hormone therapy. Supporting this hypothesis, a different study has previously shown that CEACAM6, is significantly overexpressed in tamoxifen resistant breast tumors that subsequently relapse, and stable silencing of the CEACAM6 gene, partially restores hormone sensitivity in model systems, in vitro (30). Therefore, CEACAM19, similarly to $C E A C A M 6$, may represent a novel therapeutic target, in certain subgroups of $\mathrm{BC}$ patients, for example those who are ER-negative. Besides, our results revealed that breast tumors derived from premenopausal women, that are known to have poorer outcome, as well as more aggressive tumors (35), were significantly $(\mathrm{p}=0.016)$ more frequently found to be CEACAM19-positive, compared to those obtained from postmenopausal women.

In conclusion, our data demonstrate that significantly higher CEACAM19 expression levels are found in breast tumors compared to their corresponding normal counterparts. Moreover, CEACAM19 expression status is associated with tumor grade and Ki67 proliferative index and negatively related to ER status and patients' menopausal state. Therefore, our overall findings provide the first evidence that CEACAM19 expression is associated with certain clinicopathological features indicative of poor prognosis in $\mathrm{BC}$ patients and suggest that CEACAM19, in combination with other established markers, may serve as a valuable tool in the early diagnosis and prognosis of BC. A large scale clinical study, incorporating patient follow-up data, is our main future goal in 
order to further strengthen the clinical value of this promising biomarker.

\section{Acknowledgements}

This study was carried out with the financial support of the Commission of the European Community through the INsPiRE project (EU-FP7-REGPOT-2011-1, proposal 284460).

\section{References}

1. Jemal A, Bray F, Center MM, Ferlay J, Ward E and Forman D: Global cancer statistics. CA Cancer J Clin 61: 69-90, 2011.

2. Weigelt B, Peterse JL and van't Veer LJ: Breast cancer metastasis: markers and models. Nat Rev Cancer 5: 591-602, 2005.

3. Bogenrieder T and Herlyn M: Axis of evil: molecular mechanisms of cancer metastasis. Oncogene 22: 6524-6536, 2003.

4. Kuespert K, Pils S and Hauck CR: CEACAMs: their role in physiology and pathophysiology. Curr Opin Cell Biol 18: 565-571, 2006

5. Obrink B: CEA adhesion molecules: multifunctional proteins with signal-regulatory properties. Curr Opin Cell Biol 9: 616-626, 1997.

6. Gray-Owen SD and Blumberg RS: CEACAM1: contact-dependent control of immunity. Nat Rev Immunol 6: 433-446, 2006.

7. Huang J, Hardy JD, Sun Y and Shively JE: Essential role of biliary glycoprotein (CD66a) in morphogenesis of the human mammary epithelial cell line MCF10F. J Cell Sci 112: 4193-4205, 1999.

8. Horst AK and Wagener C: CEA-Related CAMs. Handb Exp Pharmacol, pp283-341, 2004

9. Nittka S, Gunther J, Ebisch C, Erbersdobler A and Neumaier M The human tumor suppressor CEACAM1 modulates apoptosis and is implicated in early colorectal tumorigenesis. Oncogene 23: 9306-9313, 2004.

10. Singer BB, Scheffrahn I and Obrink B: The tumor growth-inhibiting cell adhesion molecule CEACAM1 (C-CAM) is differently expressed in proliferating and quiescent epithelial cells and regulates cell proliferation. Cancer Res 60: 1236-1244, 2000

11. Duxbury MS, Ito H, Zinner MJ, Ashley SW and Whang EE: CEACAM6 gene silencing impairs anoikis resistance and in vivo metastatic ability of pancreatic adenocarcinoma cells Oncogene 23: 465-473, 2004.

12. Blumenthal RD, Leon E, Hansen HJ and Goldenberg DM: Expression patterns of CEACAM5 and CEACAM6 in primary and metastatic cancers. BMC Cancer 7: 2, 2007.

13. Singer BB, Scheffrahn I, Kammerer R, Suttorp N, Ergun S and Slevogt H: Deregulation of the CEACAM expression pattern causes undifferentiated cell growth in human lung adenocarcinoma cells. PLoS One 5: e8747, 2010.

14. Chevinsky AH: CEA in tumors of other than colorectal origin. Semin Surg Oncol 7: 162-166, 1991.

15. Chan $\mathrm{CH}$ and Stanners CP: Recent advances in the tumour biology of the GPI-anchored carcinoembryonic antigen family members CEACAM5 and CEACAM6. Curr Oncol 14: 70-73, 2007.

16. Hostetter RB, Campbell DE, Chi KF, et al: Carcinoembryonic antigen enhances metastatic potential of human colorectal carcinoma. Arch Surg 125: 300-304, 1990.

17. Blumenthal RD, Hansen HJ and Goldenberg DM: Inhibition of adhesion, invasion, and metastasis by antibodies targeting CEACAM6 (NCA-90) and CEACAM5 (Carcinoembryonic Antigen). Cancer Res 65: 8809-8817, 2005.
18. Jantscheff P, Terracciano L, Lowy A, et al: Expression of CEACAM6 in resectable colorectal cancer: a factor of independent prognostic significance. J Clin Oncol 21: 3638-3646, 2003.

19. Ballesta AM, Molina R, Filella X, Jo J and Gimenez N: Carcinoembryonic antigen in staging and follow-up of patients with solid tumors. Tumour Biol 16: 32-41, 1995.

20. Scorilas A, Chiang PM, Katsaros D, Yousef GM and Diamandis EP: Molecular characterization of a new gene, CEAL1, encoding for a carcinoembryonic antigen-like protein with a highly conserved domain of eukaryotic translation initiation factors. Gene 310: 79-89, 2003.

21. Weigel MT and Dowsett M: Current and emerging biomarkers in breast cancer: prognosis and prediction. Endocr Relat Cancer 17: R245-R262, 2010

22. Harris L, Fritsche H, Mennel R, et al: American Society of Clinical Oncology 2007 update of recommendations for the use of tumor markers in breast cancer. J Clin Oncol 25: 5287-5312, 2007.

23. Henderson IC and Patek AJ: The relationship between prognostic and predictive factors in the management of breast cancer. Breast Cancer Res Treat 52: 261-288, 1998.

24. Bertos NR and Park M: Breast cancer - one term, many entities? J Clin Invest 121: 3789-3796, 2011

25. Kinsel LB, Szabo E, Greene GL, Konrath J, Leight GS and McCarty KS Jr: Immunocytochemical analysis of estrogen receptors as a predictor of prognosis in breast cancer patients: comparison with quantitative biochemical methods. Cancer Res 49: 1052-1056, 1989.

26. Livak KJ and Schmittgen TD: Analysis of relative gene expression data using real-time quantitative PCR and the 2(-Delta Delta C(T)) method. Methods 25: 402-408, 2001.

27. Camp RL, Dolled-Filhart M and Rimm DL: X-tile: a new bioinformatics tool for biomarker assessment and outcome-based cut-point optimization. Clin Cancer Res 10: 7252-7259, 2004.

28. Goldhirsch A, Wood WC, Coates AS, Gelber RD, Thurlimann B and Senn HJ: Strategies for subtypes - dealing with the diversity of breast cancer: highlights of the St. Gallen International Expert Consensus on the Primary Therapy of Early Breast Cancer 2011. Ann Oncol 22: 1736-1747, 2011.

29. Poola I, Shokrani B, Bhatnagar R, DeWitty RL, Yue Q and Bonney G: Expression of carcinoembryonic antigen cell adhesion molecule 6 oncoprotein in atypical ductal hyperplastic tissues is associated with the development of invasive breast cancer. Clin Cancer Res 12: 4773-4783, 2006.

30. Maraqa L, Cummings M, Peter MB, et al: Carcinoembryonic antigen cell adhesion molecule 6 predicts breast cancer recurrence following adjuvant tamoxifen. Clin Cancer Res 14: 405-411, 2008

31. Gaur S, Shively JE, Yen Y and Gaur RK: Altered splicing of CEACAM1 in breast cancer: identification of regulatory sequences that control splicing of CEACAM1 into long or short cytoplasmic domain isoforms. Mol Cancer 7: 46, 2008.

32. Zhou J,Zhang L, Gu Y, et al: Dynamic expression of CEACAM7 in precursor lesions of gastric carcinoma and its prognostic value in combination with CEA. World J Surg Oncol 9: 172, 2011.

33. Ilantzis C, DeMarte L, Screaton RA and Stanners CP: Deregulated expression of the human tumor marker CEA and CEA family member CEACAM6 disrupts tissue architecture and blocks colonocyte differentiation. Neoplasia 4: 151-163, 2002.

34. Serra S, Asa SL, Bamberger AM, Wagener C and Chetty R: CEACAM1 expression in pancreatic endocrine tumors. Appl Immunohistochem Mol Morphol 17: 286-293, 2009.

35. Fredholm H, Eaker S, Frisell J, Holmberg L, Fredriksson I and Lindman H: Breast cancer in young women: poor survival despite intensive treatment. PLoS One 4: e7695, 2009. 